\title{
Improvement of the Fabrication Accuracy of Fiber Tip Microoptical Components via Mode Field Expansion
}

\author{
Albertas ŽUKAUSKAS ${ }^{* 1}$, Vasileia MELISSINAKI ${ }^{* 2}$, Dalia KAŠKELYTË ${ }^{* 1}$, \\ Maria FARSARI*2 and Mangirdas MALINAUSKAS*1 \\ 1*Department of Quantum Electronics, Vilnius University, Sauletekio Ave. 9, LT-10222 Vilnius, Lithuania \\ $2^{*}$ Institute of Electronic Structure and Laser, Foundation of Research and Technology Hellas, N. Plastira 100, 70013 Heraklion, \\ Greece \\ mangirdas.malinauskas@ff.vu.lt
}

\begin{abstract}
A novel method to overcome the problem of accurately centering microoptical components built by direct laser writing on the tip of a single mode optical fiber is presented, and it includes employing mode field expansion. A hybrid microoptical component consisting of an aspherical and a conical lens integrated into a single monolithic element has been fabricated at the end-face of a fiber using the technique of direct laser writing of photopolymers. The optical performance of the fabricated microstructures has been measured experimentally. We show that optical properties of Gaussian and Bessel beams can be easily tuned by adjusting the geometrical parameters of fiber-tip microoptical elements. In addition, we experimentally demonstrate the increase of propagation distance of a Bessel beam five-fold, using the fabricated microoptical element. This improvement in the fabrication method of fiber-tip microoptical elements can be used in the manufacturing of integrated micro-optical devices for optical tweezing, laser micromachining and long focal depth optical systems.
\end{abstract}

DOI: $10.2961 / \mathrm{jlmn} .2014 .01 .0014$

Keywords: micron scale axicon, hybrid microoptical component, fiber tip microstructure, direct laser writing, multiphoton polymerization, hybrid photopolymers.

\section{Introduction}

The idea for microoptical components (MOCs) integrated onto the tip of an optical fiber arose in 1972, when it was suggested that they could be used to increase the coupling efficiency of semiconductor lasers and optical fibers [1]. A year later, the first experimental demonstration of a spherical end-face fiber was reported, fabricated using a thermal melting technique [2]. Since then, fiber-tip MOCs have been employed in a wide spectrum of applications, ranging from the initial light coupling experiments [3] to near field imaging [4], optical sensing [5, 6], SERS [7], optical coherence tomography [8] and optical transfection [9]. However, the precise centering of the MOCs with the fiber core remains even today a challenging, albeit crucial, fabrication issue. Simple structures like conical lenses can be easily fabricated precisely centered using wet-chemical etching [10] or free-radical photopolymerization [11] techniques. In the first case, the difference in etching rates of the fiber core and cladding leads to the formation of a conical tip with base diameter equal to the core diameter. The geometrical parameters of the conical lens can be tailored by adjusting etching time, etchant concentration and solution temperature. Additionally, post-processing of the conical tip structures using fire polishing and/or arc melting allows the transformation of a conical lens to hemispherical. In order to realize more complicated MOC geometries, selective doping of the fiber is needed [12]. In the second case, when free-radical polymerization is used, the one end of the fiber is covered with a UV photosensitive material, and UV laser light is coupled into the other end. As the light emerges from the fiber, it induces photopolymerization and subsequently, an elongated spherical end-face fiber tip forms. In both cases, the MOC geometry is restricted to simple symmetrical shapes such as conical or spherical end-face waveguides, and the applications of the fiber tip structures are limited. More complex shapes and asymmetrical elements can be fabricated using direct writing techniques such as electron beam lithography (e-beam) [13], focused ion beam milling (FIB) [14], and direct laser writing (DLW) $[15,16]$. Here, the MOC centering accuracy depends on the sample positioning system. It can be defined as the ratio of the MOC deviation from the fiber core center $(\Delta l)$ and mode field diameter at the end of the fiber $\left(\omega_{0}\right): \Delta l / \omega_{0}$. Thereby, the centering can be reduced by either improving the sample positioning, thus reducing $\Delta l$, or by increasing the mode field diameter $\omega_{0}$. The latter method is more desirable in practice and has been already realized by Kim et al. [17] and Kong et al. [18]. The former spliced a coreless silica fiber to the end of the single mode fiber, whereas the latter 
used fusion splicing of a photonic crystal fiber for the mode field expansion. In both cases, the beam waist diameter of the light emerging from the fiber can be controlled by adjusting the length of the coreless silica fiber or the fusion-splicing region. In practice, this cannot be realized easily and adds an extra step in the fabrication procedure.

In this report, we present a novel method to realize beam expansion and fabrication of the MOCs on the tip of the single mode optical fiber in a single procedure. The fabrication of MOCs is done by DLW technique, a method widely used in microoptics [19, 20], photonics and metamaterials [21-23] and in biomedical applications [24-27], as it allows the fabrication of $3 \mathrm{D}$ microstructures with the sub-diffraction limit spatial resolution [28-31]. The efficiency of the new method is demonstrated with the fabrication of a monolithic fiber-end MOC to generate a Bessel beam.

\section{Experimental procedure}

Figure 1 (a) is a schematic drawing of the proposed method for improving the MOC centering accuracy exploiting the mode field expansion. Compared to other methods [17, 18], it is easier to implement, as the mode field expansion and MOC are realized in a single step. This is demonstrated by the fabrication of a hybrid monolithic MOC consisting of a conical lens, an aspherical lens and three pillars, whose purpose is to modify the Gaussian output of the fiber into a Bessel beam. The pillars serve two purposes: firstly, is to ensure the attachment of the free-form MOC to the fiber, and secondly, to control the length of the free-space between the fiber and the hybrid MOC. The light emerging from the fiber diffracts in free-space and the mode field diameter $(\omega)$ increases according to the relationship: $\omega=\omega_{0}+d \times \tan \theta$, where $\omega_{0}$ is mode field diameter at the end-face of the fiber, $d$ - the distance from the fiber and $\theta=\arcsin (\mathrm{NA} / n)-$ diffraction angle (NA is the numerical aperture, and $n$-refractive index of free-space). Consequently, the mode diameter $(\omega)$ can be easily adjusted by varying the

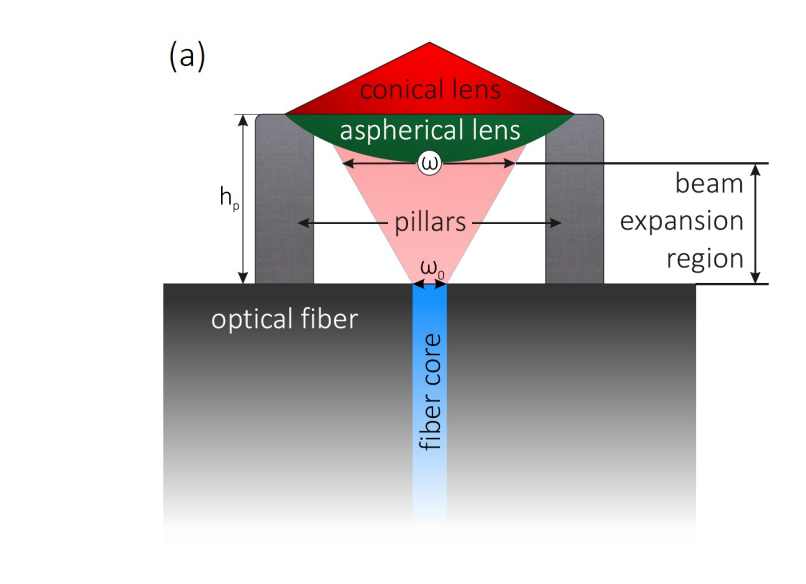

distance from the fiber $(d)$, thus the height of the pillars $\left(h_{p}\right)$. The purpose of the aspherical and conical microlenses is to ensure the collimation of the Gaussian beam and the generation of a Bessel beam, respectively. It is worth mentioning that while separate parts of the structure are sketched in different colors in Fig. 1 (a), the whole structure is in fact a monolithic component manufactured in a single step.

The procedure for fabricating the hybrid MOC on the tip of the optical fiber using DLW technology is as follows:

1. A single-mode optical fiber $\left(0.35 \mathrm{NA}, \omega_{0}=3.3 \mu \mathrm{m}\right.$, UHNA3, Thorlabs Inc.) is cut using a high precision diamond blade cleaver and cleaned with methanol to remove impurities and contaminants.

2. A organic-inorganic photopolymer (SZ2080, IESLFORTH [32]) is drop-cast on a glass substrate and heated for 5 minutes at $70^{\circ} \mathrm{C}$, to gelate.

3. The fiber tip is immersed into the photopolymer droplet. The distance between the glass substrate and the fiber tip is controlled with a micrometer positioning stage. The precise control of this distance enables repeatable and high accuracy fabrication. After immersion, sample is left for 24 hours at ambient conditions, for any solvents to evaporate.

4. The MOC is fabricated on the fiber tip. The experimental setup is shown in Fig. 1 (b) and is described in detail in [33]. In this case, the light is the second harmonic of an Yb:KGW laser ( $\lambda=515 \mathrm{~nm}, \tau=300 \mathrm{fs}, f=200 \mathrm{kHz}$, "Pharos", Light Conversion Ltd.), tightly focused with a high NA objective (1.4NA, 63×). An illumination unit consisting of a semiconductor laser and a focusing lens has been integrated into the fabrication setup for the centering of the fiber. The laser light is coupled into the one end of the fiber and the light emerging from the fiber core at the other end is imaged using a CMOS camera. The

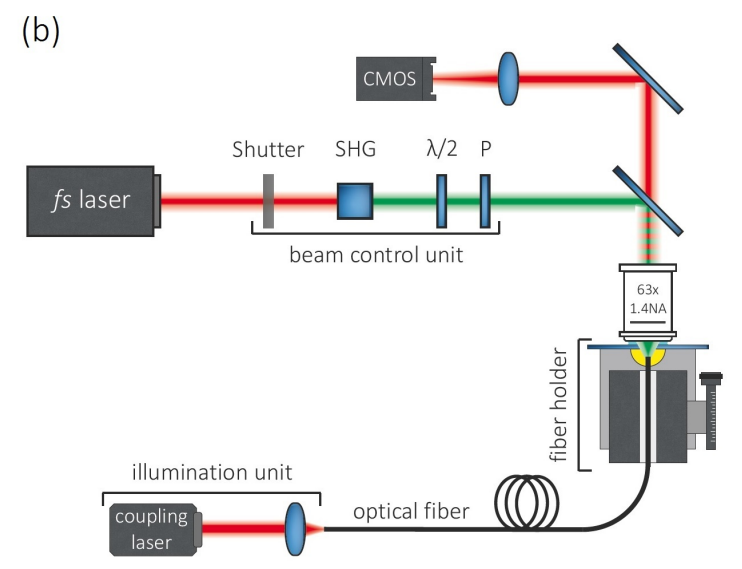

Fig. 1: (a) Schematic drawing of the proposed method to overcome the problem of accurately centering microoptical components exploiting mode field expansion. (b) Experimental setup of the direct laser writing technique for the fabrication of fiber tip microoptical components. 
fiber core is aligned with the focused writing laser beam by translating the fiber holder using linear motion stages (AEROTECH assembled by Altechna R\&D). Using this method, the maximum centering deviation is $\sim 600 \mathrm{~nm}$.

5. The unexposed material is removed by immersing the sample in 4-methyl-2-pentanone (Sigma Aldrich) for 1 hour. After this the final 3D microstructure is revealed.

The geometrical properties of the fiber tip hybrid MOCs were determined with scanning electron microscopy (SEM, TM1000, Hitachi). The optical characterization was carried out using a custom-built optical setup [34]. A He-Ne laser $(\lambda=632.8 \mathrm{~nm})$ was coupled into the one fiber end, and the light emerging from the other fiber end was imaged at various distances using a CCD camera (WinCamD- UCD15, DataRay Inc.) attached to a microscope objective $(0.65 \mathrm{NA}, 40 \times)$. Afterwards, the intensity distribution of the captured images are fitted with a Gaussian or Bessel function and the beam waist dependence on the propagation distance from the fiber tip was calculated.

\section{Results and discussion}

\subsection{Fabrication of fiber tip microoptical components}

The MOC dimensions depend on the beam waist diameter, which is a function of the distance from the fiber, and its numerical aperture (in our case NA $=0.35$ ). The mode field diameter dependence on the distance from the fiber end-face was measured firstly. Results are shown in Fig. 2. As one can observe, the beam waist increases linearly with the increase of the distance. The dimensions of the aspherical and conical lenses were determined from this graph.

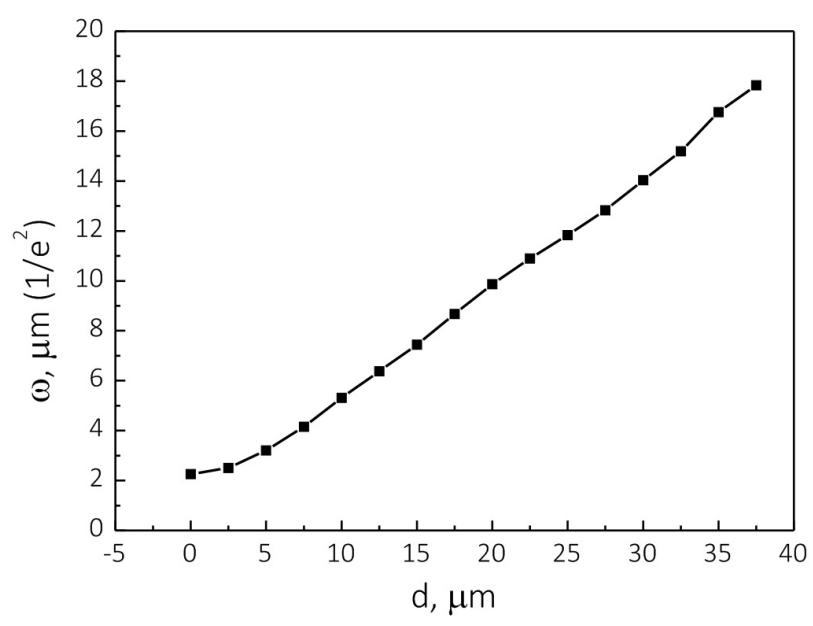

Fig. 2: Beam waist dependence on the propagation distance from the fiber tip through single mode optical fiber.

Figure 3 (a) shows a tilted SEM image of the cleaved singlemode optical fiber. It can be seen that the cut is of good quality and there is no obvious damage to the fiber core and cladding. Figure 3 (b) shows an image of an aspherical lens built on three triangular pillars, whose center is $23 \mu \mathrm{m}$ apart from the fiber center; the purpose of this MOC is to collimate the laser beam. To help the reader, the aspherical lens is colored green. To obtain a collimated beam, the pillar height $h_{p}$ needs to be the same as the aspherical lens focal distance $f$; in this case $35 \mu \mathrm{m}$. The radius $R$ and lens thickness $h_{\text {asp }}$ were set to $20 \mu \mathrm{m}$ and $10.54 \mu \mathrm{m}$, respectively. Using SEM, it was possible to measure the actual dimensions of the fabricated MOC, which were: $h_{p}=34.44 \pm 0.39 \mu \mathrm{m}, R=19.48 \pm 0.16 \mu \mathrm{m}$ and $h_{\text {asp }}=11.04 \pm 0.39 \mu \mathrm{m}$. This structure was built using a concentric ring fabrication algorithm with $50 \mathrm{~nm}$ lateral spacing between two adjacent rings, scanning velocity of $50 \mu \mathrm{m} / \mathrm{s}$ and intensity of $0.274 \mathrm{TW} / \mathrm{cm}^{2}$ (measured before the objective). The deviation of the aspherical lens center from the fiber core center was also measured and was found to be $\sim 600 \mathrm{~nm}$.

Figure 3 (c) shows an SEM image of the hybrid MOC described earlier in Fig. 1 (a). Here, the conical lens is colored red. The radius and cone angle $(\beta)$ of the conical lens was set to $20 \mu \mathrm{m}$ and $150^{\circ}$, respectively. For structuring this MOC, the laser beam scanning speed was $250 \mu \mathrm{m} / \mathrm{s}$ and the laser intensity $0.329 \mathrm{TW} / \mathrm{cm}^{2}$. Figure 3 (d) shows a tilted SEM image of the hybrid MOC. Here the fiber core is clearly visible as all the unpolymerized material was successfully removed.
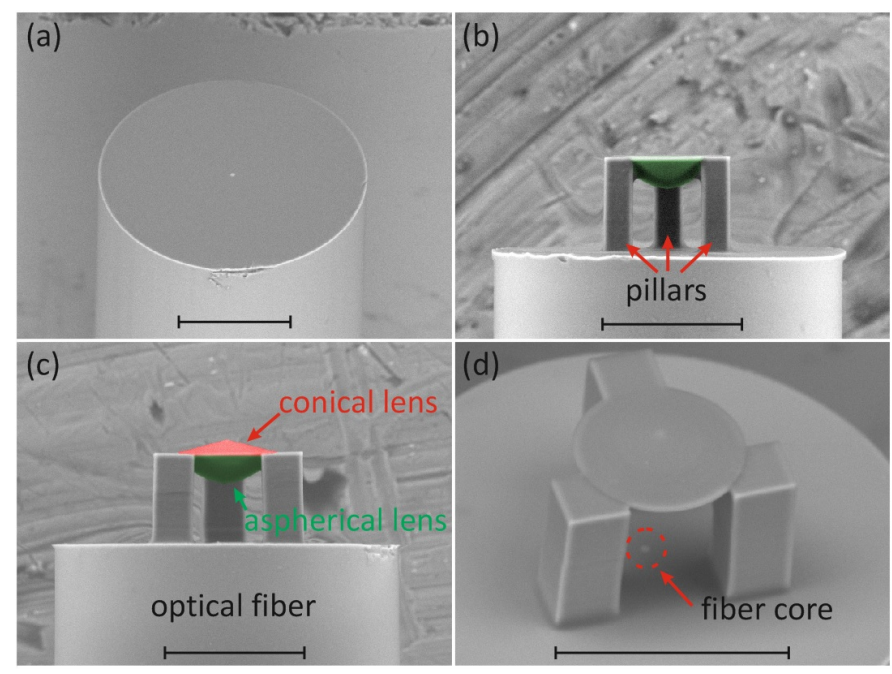

Fig. 3: SEM images of the cleaved single mode optical fiber (a), aspherical lens (b) and hybrid microoptical element (c) fabricated on the tip of the fiber. Tilted SEM image of the MOC structure is shown in (d). Scale bar: $50 \mu \mathrm{m}$.

\subsection{Optical characterization of fiber tip microoptical com- ponents}

Figure 4 shows the beam waist dependence on the propagation distance from the base of the aspherical lens of Fig. 3 (b). It was expected to obtain a collimated beam, i.e. $\omega=$ constant over the whole propagation distance. It can be seen that after $50 \mu \mathrm{m}$ travel, the beam waist has increased by $2.21 \mu \mathrm{m}(4 \%)$. This small deviation is most likely caused due to the pillars being shorter $\left(h_{p}=34.44 \pm 3.9 \mu \mathrm{m}\right.$,) than the theoretical value $\left(h_{p}=35 \mu \mathrm{m}\right)$, as the condition $h_{p}=f$ is not fulfilled. 


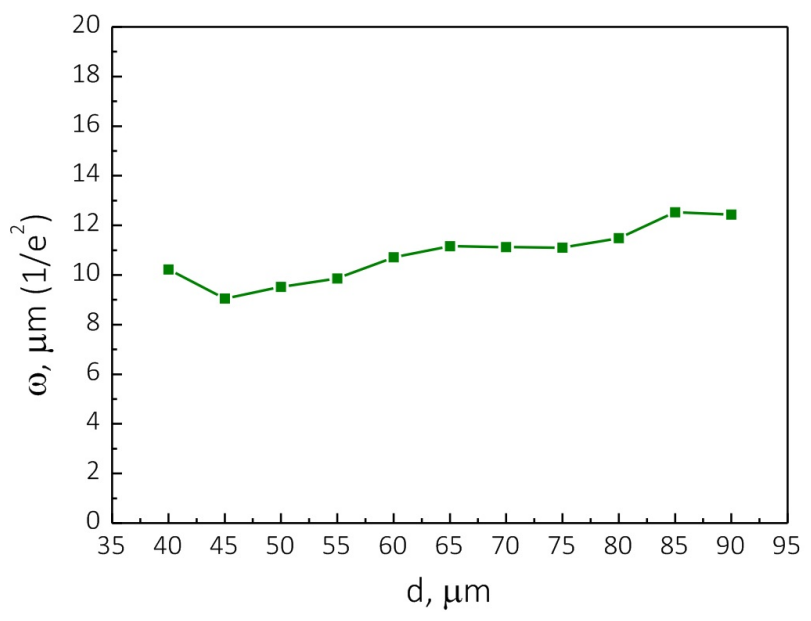

Fig. 4: Beam waist dependence on the propagation distance through aspherical lens fabricated on the fiber tip.

Figure 5 shows the beam waist diameter as a function of the propagation distance from the tip of the conical lens. Conical lenses are widely used for the efficient transformation of Gaussian beams to Bessel beams. It can be seen that the generated Bessel beam diffracts $5.32 \mu \mathrm{m}$ after $75 \mu \mathrm{m}$; this error is probably due to the non-perfect collimation of the original Gaussian beam. The inset in Fig. 5 shows the transverse intensity distribution at the maximum intensity plane from the conical lens tip (marked with arrow in Fig. 5). It can be seen that, despite the deviation from the optimum, the beam exhibits a Bessel- like intensity distribution.

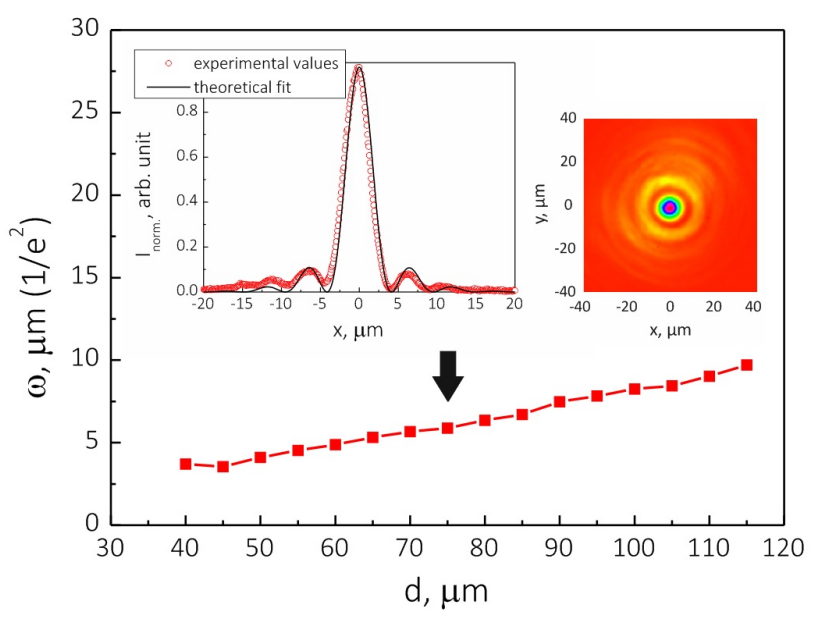

Fig. 5: Beam waist dependence on the propagation distance through hybrid MOC fabricated on the fiber tip. Inset shows transverse intensity distribution of the Bessel beam and $2 D$ intensity profile.

Let us evaluate the influence of the beam expansion to the reduction of the centering problem. Centering accuracy can be defined as $\varphi=[\Delta l / \omega] \times 100 \%$, where $\Delta l$ is the deviation from the center of the fiber and $\omega$ is the beam waist. $\varphi=0 \%$ means that the centers of the fiber core and MOC coincide perfectly. It was found that $\Delta l=600 \mathrm{~nm}, \omega_{0}=2.26 \mu \mathrm{m}$ and $\omega=16.76 \mu \mathrm{m}$ according to measured experimental results (here $\omega_{0}$ and $\omega$ are the beam waist at the end-face of the fiber and at $35 \mu \mathrm{m}$ distance from the fiber, respectively). Subsequently, $\Delta l / \omega$ equals $\varphi_{0} \simeq 27 \%$ and $\varphi \simeq 4 \%$, respectively. The beam expansion, therefore, reduces the relative off-axis error 7 -fold.

In addition to reducing the accurate centering problem, mode field expansion allows much longer Bessel beam propagation distances $\left(z_{\max }\right)$. It is known that $z_{\max }$ depends linearly on the incident beam waist $\omega_{0}$ according to the relationship:

$$
z_{\max }=\frac{\omega_{0}}{(n-1) \gamma},
$$

where $\gamma=(180-\beta) / 2$ is the inclination angle of the conical lens surface. Here, the incident beam waist at the conical lens base is $10.22 \mu \mathrm{m}$, thus the maximum propagation distance is $z_{\text {max }}=77.7 \mu \mathrm{m}$ (measured $z_{\max }=75 \pm 2.5 \mu \mathrm{m}$ ). In comparison, the beam waist at the fiber tip is $2.26 \mu \mathrm{m}$, thus $z_{\max }=17.13 \mu \mathrm{m}$. Consequently, the distance has increased by 4.5 times and it is limited only by the fiber numerical aperture.

\section{Conclusion}

In conclusion, we have proposed a new method to overcome the problem of accurately centering microoptical components fabricated using direct laser writing on the tip of a single mode optical fiber by employing mode field expansion. We have used this method to fabricate a hybrid microoptical component consisting of an aspherical and a conical lens integrated into a single monolithic element at the end-face of a fiber. The optical performance of the fabricated microstructures has been measured experimentally. We have shown that the optical properties of Gaussian and Bessel beams can be easily tuned by adjusting the geometrical parameters of the MOC. In addition, we experimentally demonstrated the increase of propagation distance of a Bessel beam by 5 times, using the fabricated MOC. This improvement in the fabrication method of fiber-tip microoptical elements can be used in the fabrication of integrated micro devices for optical tweezing, laser micromachining and long focal depth optical systems.

\section{Acknowledgments}

A.Ž., D.K. and M.M. acknowledge financial support by a research grant No. VP1-3.1-ŠMM-10-V-02-007 (Development and Utilization of a New Generation Industrial Laser Material Processing Using Ultrashort Pulse Lasers for Industrial Applications) from the European Social Fund Agency. V.M. and M.F. acknowledge financial support from the Thales Program 3DSET (MIS 380278).

\section{References}

[1] L.G. Cohen: Bell Syst. Tech. J., 51, (1972) 573.

[2] D. Kato: J. Appl. Phys., 44, (1973) 2756.

[3] E. Weidel: Opt. Commun., 12, (1974) 93. 
[4] Z. Sedaghat, A. Rumyantseva, A. Bruyant, S. Kostcheev, S. Blaize, S. Jradi, R. Bachelot and A. Monmayrant: Appl. Phys. Lett., 100, (2012) 1.

[5] S.K. Eah, H.M. Jaeger, N.F. Scherer, G.P. Wiederrecht and X.M. Lin: Appl. Phys. Lett., 86, (2005) 1.

[6] M. Konstantaki, P. Childs, M. Sozzi and S. Pissadakis: Laser Photonics Rev., 7, (2013) 439.

[7] E.J. Smythe. E. Cubukcu and F. Capasso: Opt. Express, 15, (2007) 7439.

[8] K.M. Tan, M. Mazilu, T.H. Chow, W.M. Lee, K. Taguchi, B.K. Ng, W. Sibbett, C.S. Herrington, C.T.A. Brown and K. Dholakia: Opt. Express, 17, (2009) 2375.

[9] N. Ma, P.C. Ashok, D.J. Stevenson, F.J. Gunn-Moore and K. Dholakia: Biomed. Opt. Express, 1, (2010) 694.

[10] G. Einsenstein and D. Vitallo: Appl. Opt., 21, (1982) 3470.

[11] R. Bachelot, C. Ecoffet, D. Deloeil, P. Royer and D.J. Lougnot: Appl. Opt., 40, (2001) 5860.

[12] S. Pevec, E. Cibula, B. Lenardic and D. Donlagic: IEEE Photon. J., 3, (2011), 627.

[13] M. Prasciolu, P. Candeloro, R. Kumar, L. Businaro, E. Di Fabrizio, D. Cojoc, S. Cabrini, C. Liberale and V. Degiorgio: Jpn. J. Appl. Phys., 42, (2003) 4177.

[14] W. Chen, W. Han, D.C. Abeysinghe, R.L. Nelson and Q. Zhan: J. Opt., 13, (2011) 1.

[15] C. Liberale, G. Cojoc, P. Candeloro, G. Das, F. Gentile, F. De Angelis and E. Di Fabrizio: Photonic. Tech. L., 22, (2010) 474.

[16] M. Malinauskas, A. Žukauskas, V. Purlys, K. Belazaras, A. Momot, D. Paipulas, R. Gadonas, A. Piskarskas, H. Gilbergs, A. Gaidukevičiūtè, I. Sakellari, M. Farsari and S. Juodkazis: J. Opt., 12 (2010) 1.

[17] K.R. Kim, S. Chang and K. Oh: IEEE Photonic. Tech. L., 15, (2003) 1100.

[18] G.J. Kong, J. Kim, H.Y. Choi, J.E. Im, B.H. Park, U.C. Peak and B.H. Lee: Opt. Lett., 31, (2006) 894.

[19] A. Žukauskas, M. Malinauskas and E. Brasselet: Appl. Phys. Lett., 103, (2013) 181122.

[20] T. Grossmann, S. Schleede, M. Hauser, T. Beck, M. Thiel, G. von Freymann, T. Mappes, H. Kalt: Opt. Express, 19, (2011) 11451.

[21] J. Trull, L. Maigytė, V. Mizeikis, M. Malinauskas, S. Juodkazis, C. Cojocaru, M. Rutkauskas, M. Peckus, V. Sirutkaitis and K. Staliūnas: Phys. Rev. A, 84, (2011) 033812 .
[22] N. Vasilantonakis, K. Terzaki, I. Sakellari, V. Purlys, D. Gray, C.M. Soukoulis, M. Vamvakaki, M. Kafesaki, M. Farsari: Adv. Mater., 24, (2012) 1101.

[23] C.M. Soukoulis, M. Wegener: Nat. Photonics, 5, (2011) 523.

[24] P. Danilevičius, S. Rèkštytè, E. Balčiūnas, A. Kraniauskas, R. Jarašienè, R. Šimeikis, D. Baltriukienė, V. Bukelskienè, R. Gadonas and M. Malinauskas: J. Biomed. Opt., 17, (2012) 081405.

[25] K. Terzaki, E. Kalloudi, E. Mossou, E.P. Mitchell, V.T. Forsyth, E. Rosseeva, P. Simon, M. Vamvakaki, M. Chatzinikolaidou, A. Mitraki, M. Farsari: Biofabrication, 5, (2013) 045002.

[26] J. Torgersen, X.H. Qin, Z.Q. Li, A. Ovsianikov, R. Liska, J. Stampfl: Adv. Funct. Mater., 23, (2013) 4542-4554.

[27] A. Ovsianikov, V. Mironov, J. Stampfl, R. Liska: Expert Rev. Med. Devices, 9, (2012) 613.

[28] M. Malinauskas, M. Farsari, A. Piskarskas and S. Juodkazis: Phys. Rep., 533, (2013) 1.

[29] J. Fischer, M. Wegener: Opt. Mater. Express, 1, (2011) 614.

[30] E. Kabouraki, A.N. Giakoumaki, P. Danilevičius, D. Gray, M. Vamvakaki, M. Farsari: Nano Lett., 13, (2013) 3831.

[31] R. Wollhofen, J. Katzmann, C. Hrelescu, J. Jacak, T.A. Klar: Opt. Express, 21, (2013) 10831.

[32] A. Ovsianikov, J. Viertl, B.N. Chichkov, M. Oubaha, B. MacCraith, I. Sakellari, A. Giakoumaki, D. Gray. M. Vamvakaki, M. Farsari and C. Fotakis: ACS Nano, 2, (2008) 2257.

[33] M. Malinauskas, G. Kiršanskè, S. Rekštytė, T. Jonavičius, E. Kaziulionytė, L. Jonušauskas, A. Žukauskas, R. Gadonas and A. Piskarskas: Lith. J. Phys., 52, (2012) 312.

[34] M. Malinauskas, H. Gilbergs, A. Žukauskas, V. Purlys, D. Paipulas and R. Gadonas: J. Opt., 12, (2010) 035204.

(Received: July 22, 2013, Accepted: February 19, 2014) 